\title{
La comunicación en los equipos de salud
} Communication in health care teams

\author{
Dr. Juan B. Dartiguelongue ${ }^{a, b, c}$ y Dr. Pablo J. Cafiero ${ }^{b, d}$
}

\section{RESUMEN}

Una característica esencial de las instituciones de salud es el trabajo en equipo, el cual implica una filosofía organizacional donde el talento colectivo, con objetivos comunes que trazan direcciones claramente identificadas, posibilita mejores resultados. La comunicación ocupa un lugar central en este modelo, entendido como un proceso de interacción y no solo una actividad destinada a transmitir información. El error médico y el conflicto en el ámbito institucional son causados, frecuentemente, por fallas en la comunicación efectiva. Aligual que en otras áreas de la salud, los aspectos comunicacionales del trabajo en equipo evolucionan con el aprendizaje. La adquisición de estas competencias, el desarrollo de la escucha activa y la interacción entre disciplinas favorecen la formación profesional yla seguridad del paciente. Dehecho, junto con otros factores, estos aspectos necesarios de la comunicación ponen de relieve la calidad de atención en el campo de la salud.

Palabras clave: comunicación, grupo de atención al paciente, comunicación interdisciplinaria.

http:/ / dx.doi.org/10.5546/aap.2021.e589

Texto completo en inglés:

http:/ / dx.doi.org/10.5546/aap.2021.eng.e589

Universidad de

Buenos Aires,

Argentina.

c. Sociedad Argentina de Pediatría, Región Metropolitana,

Ciudad Autónoma de Buenos Aires, Argentina.

d. Hospital de Pediatría S.A.M.I.C "Prof. Dr. Juan P. Garrahan", Ciudad Autónoma de Buenos Aires, Argentina.

Correspondencia:

Dr. Juan B.

Dartiguelongue:

jbdartiguelongue@

hotmail.com

Financiamiento:

Ninguno.

Conflicto de intereses: Ninguno para declarar.

Recibido: 12-1-2021

Aceptado: 10-6-2021
Cómo citar: Dartiguelongue JP, Cafiero PJ. La comunicación en los equipos de salud. Arch Argent Pediatr 2021;119(6):e589-e593.

\section{INTRODUCCIÓN}

La comunicación es un fenómeno inherente al ser humano. Involucra a dos o más personas entre las que circulan mensajes a través de diferentes vías, en un contexto espacial e histórico, estableciendo significados e inculcando creencias. Este fenómeno, tan cotidiano como particular, modela las posibilidades de comprensión y, por lo tanto, define las relaciones humanas. ${ }^{1,2}$ Representa un acto de influencia recíproca, en el cual cada sujeto modifica su comportamiento en reacción al otro. ${ }^{3}$ construye la realidad, pues sustenta el desarrollo de la sociedad, el arte y
Mediante la comunicación se la cultura, al tiempo que fundamenta el consenso, el disenso y la empatía entre personas.

Aun en ausencia de palabras, la comunicación es constante e ininterrumpida. Las posiciones corporales, las expresiones faciales, la respiración, la mímica y las gesticulaciones amplifican los canales de comunicación que permiten la interpretación del sujeto en base a su estado de ánimo, sentimientos y carácter.

La comunicación es efectiva siempre y cuando reasegure la compresión del mensaje en todas sus dimensiones. ${ }^{4}$ No resulta extraño que sea reconocida como uno de los factores centrales que sustentan la seguridad y la calidad del trabajo, particularmente en equipos que desempeñan actividades complejas. ${ }^{5}$ En el ámbito de la salud, la efectividad de la comunicación ocupa un lugar central como un proceso fundamentado en la interacción, lejos de significar una actividad meramente destinada a transmitir información. ${ }^{6}$

Los modelos que revelan la comunicación desde una perspectiva que pondera la relación entre seres humanos conciben la transmisión de mensajes de forma helicoidal, a diferencia de la representación lineal del modelo mecanicista clásico. Estos modelos resultan adecuados para definir a la comunicación en salud, donde además de transmitir mensajes lo suficientemente inteligibles, se tiene en consideración a quien los recibe para la comprensión e interpretación de su significado. ${ }^{1}$

Por mucho tiempo, la investigación en comunicación estuvo basada en la presencia de la palabra, en lo que se dice o deja de decir. Sin embargo, la importancia del silencio es evidente 
para cualquiera que integre o haya integrado equipos de salud, en particular cuando se trata de silencios funcionales o significativos. ${ }^{7}$

La calidad de la atención médica depende directamente del funcionamiento de los equipos de trabajo y, por lo tanto, este intercambio profesional efectivo funciona como el engranaje del sistema. ${ }^{8}$ La forma en que se comunica es tan importante como el contenido, por esta razón saber comunicar es una habilidad básica y relevante. Asimismo, estas competencias son un pilar fundamental en la formación médica y en la construcción de la relación médicopaciente, aspectos del trabajo en equipo que pueden ser aprendidos, con la mira en la seguridad y el bienestar del paciente y su familia. ${ }^{8-10}$ Por otra parte, el desarrollo de habilidades comunicacionales representa uno de los factores preventivos para el desarrollo del síndrome de desgaste profesional (burn out), que invariablemente tiene impacto sobre la calidad de la atención.

\section{COMUNICACIÓN, EQUIPOS DE TRABAJO Y SEGURIDAD DEL PACIENTE}

Un equipo se define como dos o más personas que tienen funciones específicas, realizan tareas interdependientes, son adaptables y comparten un objetivo común. El trabajo en equipo no se reduce a trabajar en conjunto, sino que implica una filosofía organizacional sobre el aprovechamiento del talento colectivo. Para tal fin, se requiere que los profesionales posean habilidades y aptitudes para comunicar, con diferentes trasfondos, con el propósito común que conduce hacia objetivos claramente identificados. ${ }^{11,12}$

Trabajar de manera individual, con escasa e inefectiva comunicación dentro y entre los equipos que conforman una institución de salud, es uno de los principales impedimentos para generar una atención segura y de calidad. ${ }^{5}$ De hecho, más de la mitad de los daños graves y fallecimientos se deben al fracaso en la comunicación del trabajo en equipo, con el paciente y su familia. ${ }^{13-15}$

Frente a esta situación, las instituciones deben promover un cambio cultural en el abordaje del error, fundamentado en la comunicación, cuyo horizonte sea promover las competencias respectivas entre los equipos, los pacientes y sus familias. ${ }^{16}$

Se pueden implementar diversas estrategias para identificar y corregir las debilidades y reducir los errores, dado que ciertos escenarios son particularmente proclives para la comunicación inefectiva. Tal es el caso de los pases de guardia, las interconsultas, la confección de indicaciones médicas y el traslado de pacientes dentro de las instituciones, entre otros. ${ }^{17} \mathrm{La}$ evaluación sistemática en estos espacios mediante la confección de listas de cotejo y simulaciones, entre otras herramientas, resulta imprescindible para mejorar la calidad de la atención y, por ende, la seguridad del paciente, ya que permite medir el impacto en torno al error y detectar los puntos débiles a partir de los cuales el error puede amplificarse. ${ }^{18-20}$

Asimismo, el desarrollo de la escucha activa en todas las instancias de trabajo, la generación de herramientas para la resolución de conflictos y la labor entre disciplinas, son elementos clave para optimizar la comunicación en el ámbito institucional.

\section{LA ESCUCHA ACTIVA}

El habla es efectiva únicamente cuando produce en el otro la escucha deseada. Para lograrlo es requisito no solo oír la palabra del otro, sino atender a lo que se expresa. Es la manifestación más evidente de la capacidad de relación interpersonal y de comprender al otro en su entorno. Implica escuchar con atención no solo lo que se dice, sino también lo que se pretende decir, lo que se expresa corporalmente y su significado. ${ }^{21}$ Permite comprender el contexto $y$ el lenguaje no verbal, percibir emociones y mejorar la información. A su vez, responde al interés sobre la narrativa ajena, evitando hipótesis precoces o indicios erróneos y propicia aquello que se quiere inducir en la otra persona. ${ }^{4,21}$

Lograr la escucha activa requiere distintos abordajes. Por un lado, la no anticipación al mensaje del otro - prejuicio-, junto con facilitar el relato, asentir, invitar a continuar, clarificar, pedir ejemplos, compartir pensamientos, otorgar silencios funcionales y demostrar empatía favorecen el discurso y acrecientan la capacidad de comprensión. ${ }^{3}$

Muchas veces, la necesidad de respuestas inmediatas, el escaso aprovechamiento de los recursos lingüísticos y paralingüísticos, el apremio con que deben tomarse ciertas decisiones y la realización simultánea de tareas imposibilitan la escucha activa. Es claro que, en estos escenarios, el tiempo que se destina a la comunicación resulta insuficiente, ${ }^{22}$ y junto con la falta de interés por la narrativa ajena, quizás por agobio o agotamiento profesional, ocasionan quiebres 
en la comunicación, origen del conflicto entre profesionales dentro de una institución. ${ }^{23}$

\section{EL CONFLICTO Y LA NEGOCIACIÓN}

El conflicto es un fenómeno propio entre los seres humanos, frecuentemente generado por fallas en la comunicación interpersonal. Suele tener un origen bilateral y se nutre de fenómenos que subyacen -tales como recuerdos, experiencias, antipatías y prejuicios- y afectan la transferencia y la proyección del mensaje. ${ }^{24}$ Puede estar influenciado por deseos, estados de ánimo, interpretaciones y expectativas. No es infrecuente que numerosos juicios sobre el otro estén basados en creencias o sucesos que no han sido verificados. Estos supuestos van moldeando las posibilidades de comprensión, limitan las interacciones y restringen la comunicación efectiva. ${ }^{24-26}$

La imagen de uno mismo y en qué medida se conoce al otro pueden ser el origen de los problemas de comprensión mutua. Por ejemplo, las personas o grupos con imagen negativa sobre sí mismos tienden a tomar ciertos mensajes como ataques personales, mientras que, cuanto mejor se conoce a un grupo o a una persona, más fácil es interpretar lo que dice. Sin embargo, la comunicación de los profesionales de la salud frecuentemente se establece con personas no conocidas -el paciente, su familia, otros profesionales- y esto de ninguna manera debe invalidar el diálogo ni limitar la capacidad de compresión y empatía.

La resolución del conflicto puede darse sobre diferentes estilos de negociación. En la forma competitiva el conflicto domina la escena, y lo que gana uno lo pierde el otro. La satisfacción de los intereses primordiales de cada parte excluye la posibilidad de satisfacción de los intereses de la otra; representa un estilo duro con posiciones extremas. Por lo general, se produce cuando hay ausencia de confianza entre grupos, con escasez de vínculo y objetivos comunes, que sustentan el poco interés y la distancia. ${ }^{27}$

La negociación colaborativa, en cambio, intenta satisfacer los intereses prioritarios de cada una de las partes, luego de un proceso equilibrado de análisis cuidadoso de actitudes y posibilidades de resolución. Es decir, se busca el acercamiento al ideal de que ambas partes logren el acuerdo deseado. Los intereses prioritarios de cada uno, entendidos como las necesidades, intenciones y deseos -razones por las que se quiere o se anhela algo- pueden ser comunes, diferentes u opuestos. En base a esto, se traza la posición individual o grupal, que ubica una estrategia en el campo del conflicto. Es decir, lo que cada persona o grupo manifiesta que quiere lograr de acuerdo con la narrativa que sustenta la identidad grupal o individual. Aclarar los intereses de las partes y construir nuevas posiciones sustentables son aspectos fundamentales en este modelo de negociación. Surge entonces la relevancia de concentrarse en los intereses y no en las posiciones ${ }^{27}$ dejando de lado el ego profesional y focalizando el interés en el paciente y su familia.

\section{MULTIDISCIPLINA, INTERDISCIPLINA Y TRANSDISCIPLINA.}

La naturaleza pluridisciplinaria de la atención médica, caracterizada por el accionar de diferentes equipos de profesionales de la salud, supone una forma de trabajo colaborativa que intenta alcanzar el mejor nivel de atención. En este escenario, resulta fundamental integrar los mensajes de los diferentes especialistas y profesionales de otras disciplinas que, en ocasiones, intervienen en la asistencia desde perspectivas parciales más focalizadas. $^{3}$

Para el paciente y su familia, la experiencia de un padecimiento incluye su perspectiva y forma de entender lo que ocurre, el significado que le otorga, su cultura y su contexto. Del mismo modo, el profesional y el equipo del que forma parte, también se enmarcan en un contexto personal, con sus cualidades y conocimiento. Las relaciones interprofesionales suman diversas voces, perspectivas y futuras direcciones en el proceso de comunicación entre disciplinas.

Una disciplina puede definirse como la categorización organizacional del conocimiento empírico, que divide y especializa los conocimientos en los que se basan las ciencias. Tiende a ser autónoma y se encarga de delimitar las fronteras que la dividen. Utiliza su propio lenguaje y técnicas, y puede dar origen a su propia teoría.

En la interacción entre disciplinas se destacan diferentes modalidades, entre ellas la multidisciplina, la interdisciplina y la transdisciplina. ${ }^{28}$ La multidisciplina es el manejo de varias disciplinas en torno a un fenómeno que las abarca o afecta, pero por separado. Por ejemplo, en una investigación multidisciplinaria los expertos de cada disciplina trabajan sobre el tema enfocándose solo en su área de estudio y no toman en cuenta el trabajo de los demás. Termina siendo una compilación del punto de vista de 
cada disciplina sobre un tema específico. Se puede estudiar el maltrato infantil, por ejemplo, desde el punto de vista del derecho, de la sociología, de la psicología, de la medicina o incluso de la historia. La multidisciplina implica la fusión temática de disciplinas que actúan por separado, al tratar un mismo problema. ${ }^{28-30}$

La interdisciplina, en cambio, se caracteriza por la existencia de una relación recíproca entre disciplinas, que intentan identificar y resolver un mismo problema. ${ }^{28}$ Se refiere a la revelación de algo que se encuentra en la frontera común a varias disciplinas. Utiliza los métodos de una y los transfiere a otra, en un intercambio de conocimientos teóricos y prácticos. Es un modelo de cooperación, en el cual se establece un dialogo con el que interaccionan y encuentran un punto en común para fundamentar su trabajo. ${ }^{31,32}$ Es posible distinguir tres tipos de interdisciplina: ${ }^{28}$

- La interdisciplina como aplicación; por ejemplo, los métodos de imágenes aplicados al diagnóstico médico.

- La interdisciplina como epistemología; por ejemplo, los métodos de la lógica formal transferidos a la epistemología y a la metodología de la investigación.

- La interdisciplina como concepción de nuevas disciplinas; por ejemplo, la transferencia de los métodos de la química y de la física, que posibilita el desarrollo de la farmacología.

Por su parte, la transdisciplina puede definirse como el proceso de construcción del conocimiento mediante numerosos trabajos teórico-empíricos, abiertos a las tendencias heterogeneizantes consustanciales a toda realidad. Se refiere a la fundamentación o explicación de las disciplinas desde lo que construyen en común. Este enfoque se concibe como un conocimiento superior emergente, fruto de un movimiento dialéctico del pensamiento, que permite cruzar los linderos de diferentes áreas del conocimiento disciplinar y crear imágenes de la realidad más completas e integradas. ${ }^{28}$ Frente a esta orientación de la complejidad del ser humano, los procesos de salud y enfermedad se abordan desde la perspectiva de redes multicausales, donde toman cabida todos los conocimientos establecidos, ${ }^{33}$ como la epidemiología, fisiología, farmacología, medicina social, psicología, antropología y neurociencias, entre otros.

\section{CONCLUSIÓN}

El trabajo en equipo es el sostén de las instituciones de salud. Para que la atención sea segura, empática y de calidad, resulta imprescindible fomentar el desarrollo de habilidades comunicacionales. Este compromiso asegura el diálogo y la interacción entre profesionales y disciplinas, cuyo resultado repercute invariablemente sobre el bienestar del paciente y su familia.

\section{REFERENCIAS}

1. Ruiz Moral R. Comunicación clínica: principios y habilidades para la práctica. Madrid: Panamericana; 2014.

2. Clèries X. La esencia de la comunicación en educación médica. Educ Med. 2010;v13(1):25-31.

3. Rodríguez S, Vassallo Juan C. Comunicación en la atención médica. En: Sociedad Argentina de Pediatría. PRONAP. 2016; 2(1):45-74.

4. Klein ER. Effective communication with patients. Pa Nurse. 2005; 60(4):14-5.

5. Ceriani Cernadas JM. La comunicación en los equipos de atención médica: un desafío esencial para mejorar la seguridad del paciente. Arch Argent Pediatr. 2014;112(2):1145.

6. Becker C, Hunziker S. Die Wichtigkeit der Kommunikation in der Arzt-Patienten-Beziehung. Ther Umsch. 2019; 76(5):231-8.

7. Lingard L. Productive complications: emergent ideas in team communication and patient safety. Healthc Q. 2012; 15(Spec No):18-23.

8. Lee CT, Doran DM. The Role of Interpersonal Relations in Healthcare Team Communication and Patient Safety: A Proposed Model of Interpersonal Process in Teamwork. Can J Nurs Res. 2017; 49(2):75-93.

9. Deveugele M. Communication training: Skills and beyond. Patient Educ Couns. 2015; 98(10):1287-91.

10. Lifchez SD, Cooney CM, Redett RJ 3rd. The Standardized Professional Encounter: A New Model to Assess Professionalism and Communication Skills. J Grad Med Educ. 2015; 7(2):230-3.

11. Ruberton PM, Huynh HP, Miller TA, Kruse E, et al. The relationship between physician humility, physician-patient communication, and patient health. Patient Educ Couns. 2016; 99(7):1138-45.

12. Coomber P, Clavarino A, Ballard E, Luetsch K. Doctorpharmacist communication in hospitals: strategies, perceptions, limitations and opportunities. Int J Clin Pharm. 2018; 40(2):464-73.

13. Topcu I, Turkmen AS, Sahiner NC, Savaser S, Sen H. Physicians' and nurses' medical errors associated with communication failures. J PakMed Assoc. 2017; 67(4):600-4.

14. Bittner-Fagan H, Davis J, Savoy M.Improving PatientSafety: Improving Communication. FP Essent. 2017; 463:27-33.

15. Khajouei R, Abbasi R, Mirzaee M. Errors and causes of communication failures from hospital information systems to electronic health record: A record-review study. Int J Med Inform. 2018; 119:47-53.

16. Bascuñán ML, Arriagada AM. Comunicación de errores médicos a pacientes y familiares: interrogantes y herramientas. Rev Med Chil. 2016; 144(9):1185-90.

17. Ahmadipour $H, N$ ahid M. Medical error and related factors during internship and residency. Indian J Med Ethics. 2015; 12(4):215-9.

18. Fischhoff B. Evaluating science communication. Proc Natl Acad Sci U S A. 2019; 116(16):7670-5.

19. Kalet A, Pugnaire MP, Cole-Kelly K, Janicik R, et al. Teaching communication in clinical clerkships: models from the macy initiative in health communications. Acad 
Med. 2004; 79(6):511-20.

20. Ruiz-Moral R, Pérula de Torres LA. Validez y fiabilidad de un instrumento para evaluar la comunicación clínica en las consultas: el cuestionario CICAA. Aten Primaria. 2006; 37(6):320-4.

21. Kacperek L. Non-verbal communication: the importance of listening. Br J Nurs. 1997; 6(5):275-9.

22. Blackburn J, Ousey K, Goodwin E. Information and communication in the emergency department. Int Emerg Nurs. 2019; 42:30-5.

23. Brahams D, Zeitlin H. Communication between doctors and lawyers. Med Leg J. 2018; 86(2):61-2.

24. Littlejohn S, Domenici K. Engaging communication in conflict: Systemic Practice. London: SAGE Publications, Inc; 2000.

25. González-Romá V, Hernández A. Climate uniformity: its influence on team communication quality, task conflict, and team performance. J Appl Psychol. 2014; 99(6):1042-58.

26. Jameson JK. Transcending intractable conflict in health care: an exploratory study of communication and conflict management among anesthesia providers. J Health Commun. 2003; 8(6):563-81.
27. Ingouville F. Negociación Creativa, objetivos, obstáculos y soluciones. Rev La Trama. 2004;10.

28. Choi BC, Pak AW. Multidisciplinarity, interdisciplinarity and transdisciplinarity in health research, services, education and policy: 1 . Definitions, objectives, and evidence of effectiveness. Clin Invest Med. 2006; 29(6):35164.

29. Gronenborn AM. Integrated multidisciplinarity in the natural sciences. J Biol Chem. 2019; 294(48):18162-7.

30. Sluiter JK, Bos AP, Tol D, Calff M, et al. Is staff well-being and communication enhanced by multidisciplinary work shift evaluations? Intensive Care Med. 2005; 31(10):1409-14.

31. Lancaster G, Kolakowsky-Hayner S, Kovacich J, GreerWilliams N. Interdisciplinary communication and collaboration among physicians, nurses, and unlicensed assistive personnel. J Nurs Scholarsh. 2015; 47(3):275-84.

32. Fathi R, Sheehan OC, Garrigues SK, Saliba D, et al. Development of an Interdisciplinary Team Communication Framework and Quality Metrics for Home-Based Medical Care Practices. J Am Med Dir Assoc. 2016; 17(8):725-9.e10.

33. Vyt A. Interprofessional and transdisciplinary teamworkin health care. Diabetes Metab Res Rev. 2008; 24 (Suppl 1):S1069. 